\title{
HPV infection and number of lifetime sexual partners are strong predictors for 'natural' regression of CIN 2 and 3
}

\author{
JK Chan', BJ Monk ${ }^{2}$, C Brewer², KA Keefe ${ }^{2}$, K Osann ${ }^{3}$, S McMeekin², GS Rose², M Youssef ${ }^{2}$, SP Wilczynski ${ }^{4}$, \\ FL Meyskens ${ }^{2}$ and ML Berman ${ }^{*, 2}$
}

'Division of Gynecology Oncology, Department of Obstetrics and Gynecology, Stanford University Medical Center, 300 Pasteur Drive, Stanford, CA 94305, USA; '2Division of Gynecologic Oncology, Department of Obstetrics and Gynecology, Chao Family Comprehensive Cancer Center, University of California, Irvine Medical Center, 101 The City Drive, Orange, CA 92868, USA; ${ }^{3}$ Division of Hematology/Oncology, Department of Medicine, Chao Family

Comprehensive Cancer Center, University of California, Irvine Medical Center, 101 The City Drive, Orange, CA 92868, USA; ${ }^{4}$ Department of Pathology, The City of Hope National Medical Center, 1500 East Duarte Road, Duarte, CA 91010 , USA

The aim of this paper was to evaluate the factors that predict regression of untreated CIN 2 and 3. A total of 93 patients with colposcopic persistent CIN 2 and 3 lesions after biopsy were followed for 6 months. Human papillomavirus (HPV) types were determined by polymerase chain reaction at enrolment. We analysed the biologic and demographic predictors of natural regression using univariate and multivariate methods. The overall regression rate was 52\% (48 out of 93), including 58\% (22 out of 38) of CIN 2 and 47\% (26 out of 55) of CIN 3 lesions ( $P=0.3$ I for difference). Human papillomavirus was detected in $84 \%$ (78 out of 93 ) of patients. In univariate analysis, 80\% ( 12 out of I5) of lesions without HPV regressed compared to $46 \%$ (36 out of 78) of lesions with HPV infection $(P=0.016)$. Women without HPV and those who had a resolution of HPV had a four-fold higher chance of regression than those with persistent HPV (relative odds $=3.5,95 \% \mathrm{Cl}=1.4-8.6$ ). Women with five or fewer lifetime sexual partners had higher rates of regression than women with more than five partners $(P=0.003)$. In multivariate analysis, HPV status and number of sexual partners remained as significant independent predictors of regression. In conclusion, HPV status and number of lifetime sexual partners were strongly predictive of regression of untreated CIN 2 and 3.

British Journal of Cancer (2003) 89, I062-1066. doi:I0.I038/sj.bjc.660II 96 www.bjcancer.com

(c) 2003 Cancer Research UK

Keywords: high-grade cervical dysplasia; predictors; natural regression

Cervical cancer continues to be the most deadly female cancer in developing countries. (Ponten et al, 1995) The dramatic decrease in mortality rates in Western countries is attributed to cervical cancer screening. (Harlan et al, 1991) The successful implementation of these screening programmes is based on the underlying model that cervical intraepithelial lesions (CIN) behave as progressive stages of a biologic continuum towards the development of invasive cervical cancer (Richart and Barron, 1969; Campion et al, 1986).

Genital human papillomavirus (HPV) infection has been established as the critical step in the development of most cervical cancers (IARC, 1995). The cervical carcinogenesis model involves HPV infection, intraepithelial neoplasia, and invasion. Human papillomavirus infection is found in over $40 \%$ of young, sexually active, college students (Ho et al, 1998). However, the incidence of cervical cancer is uncommon compared to the lifetime cumulative incidence of viral infection. Thus, there must be essential biological mechanisms and associated risk factors other than HPV infection that are responsible for the progression of precursor lesions to invasive cervical cancer (Schiffman \& Brinton, 1995).

*Correspondence: Dr ML Berman; E-mail: mberman@uci.edu

Presented at the American College of Obstetricians and Gynecologists Annual Meeting 2002 - Donald F Richardson Award

Received 10 December 2002; revised 17 June 2003; accepted 24 June 2003
The aetiology and risk factors involved with cervical carcinogenesis are amenable to study as invasive cervical cancer typically arises after many years from a morphologically defined precancerous lesion. However, since untreated high-grade lesions can progress to invasive cancer, most women with CIN 2 or 3 are treated promptly. Accordingly, there are few reports on the natural history of high-grade dysplasia. On the other hand, not all CIN lesions progress to invasive cancer. In fact, spontaneous regression of moderate dysplasia has been reported in up to $54 \%$ of women (Nasiell et al, 1983, 1986). Since many early lesions regress without treatment, the ability to predict which lesions will regress offers the hope of avoiding treatment and its associated complications which include: cervical stenosis, incompetence, bleeding, and infection (Hillard et al, 1991; Herzog et al, 1995).

We evaluated the predictors for natural regression of untreated CIN 2 and 3 such as age, race, parity, smoking history, oral contraceptive (OCP) use, marital status, lesion grade, sexual history, and HPV status on all our patients.

\section{MATERIALS AND METHODS}

We performed an ancillary study to a prospective, double-blinded, randomized, placebo-controlled trial of $\beta$-carotene in the management of women with untreated CIN 2 or 3. Predictors for natural regression, which included age, race, parity, smoking history, OCP use, marital status, lesion grade, sexual history, and HPV status 
were prospectively analysed. Patients were recruited from the University of California, Irvine, Medical Center, nearby clinics, and colleges. Women greater than 17 years of age with biopsy-proven CIN 2 and 3 were screened. Eligible patients had colposcopic persistence of CIN 2 or 3 one month after initial biopsy and had a negative endocervical curettage. Two pathologists agreed on the cervical histologic diagnosis before enrolment. Based on the guidelines of the Institutional Review Board at University of California, Irvine, written consents were obtained from all patients.

Since many preinvasive changes can regress spontaneously, we were initially interested in studying the factors that influenced the natural regression of $\mathrm{CIN}$ in the placebo group of our trial. However, we discovered that there was no significant difference in regression rates between the $\beta$-carotene and placebo arms at the end of the study (Keefe et al, 2001). Thus, we elected to look at those factors that influenced CIN regression in all study participants assuming that all patients who regressed did so spontaneously.

\section{Diagnosis of cervical dysplasia and surveillance}

This was a 6-month ancillary study of a 2-year randomised, controlled trial of $\beta$-carotene in the management of women with untreated CIN 2 or 3 . Over the 6 months, which comprised the study period for this current report, the subjects were followed at 3 -month intervals with testing that included cytology, presence of HPV in cervical scrapings, and colposcopy. All patients underwent colposcopically-directed biopsies and endocervical curettage before treatment and at 6 months. The two study pathologists reviewed all biopsies obtained at the 6-month end point. Regression was defined as CIN 2 lesions reverting to normal or CIN 3 lesions converting to CIN 1 or normal. We defined persistent CIN 3 lesions as those that did not regress from the time of the initial biopsy to the 6-month end point. All patients with persistent CIN 3 or whose lesions progressed to CIN 3 at 6 months were taken off of the 2-year study and treated by conventional means.

\section{HPV analysis}

We collected cervical scrapings from our patients for HPV analysis at enrolment, 3 and 6 months following the initial diagnostic CIN biopsy. Persistence and resolution of HPV infection was determined by comparing the analyses between the initial and 6-month time points. We defined HPV resolution as those with a presence of HPV DNA at initial entry and subsequently had undetectable levels of any HPV DNA at the 6-month end point. Furthermore, HPV persistence was defined as those with any HPV DNA both at baseline and 6 months. Although we detected mixed HPV types in a few patients, the majority of women had one HPV type at baseline that persisted throughout the 6 months.

Polymerase chain reaction (PCR) method was used to determine HPV genotype as described (Monk et al, 1994). DNA was first amplified with $\beta$-globin primers to confirm that the DNA extracted was intact and suitable for PCR. The DNA was then amplified with MY09/MY11 consensus primers that detect about 25 mucosal HPV types as well as type-specific primers for HPV 6,16 , and 18 . The type-specific products were transferred to nylon membranes for hybridisation with ${ }^{32} \mathrm{P}$ probes. MYO9/MY11 consensus products were assigned genotypes by RFLP digestion (Bernard et al, 1994) or if necessary sequenced. If no HPV DNA was detected with the primary primer sets, the DNA was then amplified with an additional consensus primer in the E1 gene (Gregoire et al, 1989) and the product sequenced.

\section{Risk factor analysis}

We analysed the age, race, parity, smoking history, OCP use, marital status, lesion grade, sexual history, and HPV status on all our patients. We assessed the impact of various epidemiological risk factors on regression of CIN in all our patients using univariate $\left(\chi^{2}\right)$ and multivariate (logistic regression) analyses performed with Statistical Analysis Systems. All $P$-values reported are two-tailed.

\section{RESULTS}

From 1992 to 1996, 982 patients were screened for our study. We enrolled 124 women into our trial of whom 111 were eligible to be randomized to $\beta$-carotene or placebo. One hundred three patients had colposcopic persistence of CIN 2 or 3 one month after their initial biopsy. Ninety-three (90\%) patients completed our ancillary study and underwent a 6-month cervical biopsy. The 10 excluded patients did not have a documented persistence of CIN 2 or 3 after initial biopsy. The median age of our patients was 29 years (range $19-55)$. The majority (59\%) presented with CIN 3 lesions and $41 \%$ had CIN 2 disease. Approximately one-half (46/93) were Hispanic and the others were either Caucasian $(n=45)$ or Asian $(n=2)$. Fifty (54\%) women were married or have been married in the past. Twenty-four (26\%) women have completed high school or college. The median parity was one. Forty-two $(45 \%)$ women had a history of OCP use. Twenty-eight (30\%) patients were smokers and nine of these women were smoking more than one pack per day. Of the 75 women who completed the sexual history interview, $54(72 \%)$ women had their first sexual intercourse at 18 years or younger (range: 11-30) with a median of two lifetime sexual partners (range: 1-25). At enrolment, HPV infection was detected in $84 \%$ (78 out of 93) of women and most patients had HPV types that were considered high risk for progressing to cancer. The HPV types at baseline included HPV type $16(42 \%)$, type $33(12 \%)$, type $18(9 \%)$, type $31(8 \%)$, type $52(3 \%)$, type $53(3 \%)$, type $35(1 \%)$, type $39(1 \%)$, and mixed/others $(22 \%)$. Types 16,18 , and 33 were the most prevalent HPV types and 11 patients were infected with multiple HPV types.

We analysed the regression rates of CIN 2 and 3 with respect to various demographic and biological risk factors. The overall regression rate was $52 \%$ (48 out of 93 ), including $58 \%$ (22 out of 38) of CIN 2 lesions and 47\% (26 out of 55) of CIN 3 lesions $(P=0.31$ for difference between grades). Interestingly, $80 \%$ (12 out of 15) of lesions without detectable levels of HPV regressed compared to only $46 \%$ (36 out of 78) of those infected with HPV $(P=0.016)$. Of the 78 patients who initially tested positive for HPV, 24\% (19 out of 78) subsequently developed undetectable levels of HPV. Of the patients who had a resolution of their HPV infection, $63 \%$ (12 out of 19) showed regression of their disease compared to a regression rate of only $41 \%$ (24 out of 59) in those who had a persistence of their viral infection. After the study, the population was divided into two groups based on the number of lifetime sexual partners: less than or equal to five and more than five. There was a significant inverse relationship $(P=0.003)$ between number of sexual partners and probability of CIN regression (Table 1 ).

In univariate analyses, we found that absence of persistent HPV infection $(P=0.005)$, less than or equal to five sexual partners $(P=0.003)$, and Hispanic race $(P=0.05)$ were all significant predictors for regression of high-grade dysplasia. Age, parity, smoking history, OCP use, marital status, lesion grade, and age at first intercourse were not significantly associated with CIN regression (Table 1). To further examine the variables identified as important in the univariate analyses, a multivariate logistic regression analysis was performed. We confirmed that the absence of HPV infection and less than or equal to five sexual partners remained as significant independent predictors for CIN regression (Table 2). After adjusting for HPV infection and number of sexual partners, Hispanic race was no longer a significant predictor of CIN regression. Inclusion of treatment group ( $\beta$-carotene $v s$ 
Table I Univariate analyses

\begin{tabular}{|c|c|c|}
\hline Variable & $\begin{array}{c}\text { Regression rate } \\
(\%)\end{array}$ & $\begin{array}{l}\text { P-value for } \\
\text { difference }\end{array}$ \\
\hline \multicolumn{3}{|l|}{ HPV status } \\
\hline Remained negative & $80(n=15)$ & \\
\hline Resolved & $63(n=19)$ & \\
\hline Persisted & $41(n=59)$ & 0.004 \\
\hline \multicolumn{3}{|c|}{ Number of sexual partners } \\
\hline$\leqslant 5$ & 61 & \\
\hline$>5$ & 27 & 0.003 \\
\hline \multicolumn{3}{|l|}{ Race } \\
\hline Hispanic & 61 & \\
\hline White & 40 & 0.05 \\
\hline \multicolumn{3}{|l|}{ Age (years) } \\
\hline$\leqslant 30$ & 44 & \\
\hline$>30$ & 61 & 0.11 \\
\hline \multicolumn{3}{|l|}{ Parity } \\
\hline$\leqslant 2$ & 46 & \\
\hline$>2$ & 64 & 0.11 \\
\hline \multicolumn{3}{|l|}{ Smoking (ever) } \\
\hline Yes & 39 & \\
\hline No & 57 & 0.12 \\
\hline \multicolumn{3}{|l|}{ Lesion grade } \\
\hline CIN 2 & 58 & \\
\hline CIN 3 & 47 & 0.31 \\
\hline \multicolumn{3}{|c|}{ Age at first intercourse (years) } \\
\hline$<18$ & 48 & \\
\hline$\geqslant 18$ & 57 & 0.48 \\
\hline \multicolumn{3}{|c|}{ Use of oral contraceptives (ever) } \\
\hline Yes & 48 & \\
\hline No & 55 & 0.48 \\
\hline \multicolumn{3}{|l|}{ Marital status } \\
\hline Single & 49 & \\
\hline Ever-married & 54 & 0.62 \\
\hline
\end{tabular}

placebo) in the multivariate model did not change the estimates of risk of regression associated with HPV infection or number of sexual partners.

\section{DISCUSSION}

Over the last 30 years, it has been widely accepted that CIN 1, 2, and 3 represent a continuum in the development of invasive cervical cancer (Kiviat, 1996). The biology of the various CIN grades vary, however, with the majority of CIN 1 lesions regressing without treatment (Nasiell et al, 1986; Montz et al, 1992; Flannelly et al, 1994), while CIN 2 and 3 lesions have a higher risk of persisting or progressing to invasive cancer. (Luthra et al, 1987; Murthy et al, 1996) Typically, most patients with CIN 2 or 3 lesions are promptly treated with ablation of the lesion or conisation of the cervix to prevent invasive disease. Consequently, studies on the natural history of untreated CIN 2 or 3 are limited (Nasiell et al, 1983; Luthra et al, 1987; Syrjanen et al, 1992; Flannelly et al, 1994). In addition, results from previous studies of the natural history of CIN are difficult to interpret because many studies depended on cytologic evaluation rather than histologic diagnosis to confirm either diagnosis or regression of disease. Furthermore, there often was a lack of uniform criteria in assigning a grade of dysplasia among the studies (Ostor, 1993; Holowaty et al, 1999). Lastly,
Table 2 Multivariate analysis

\begin{tabular}{|c|c|c|}
\hline Variables & Odds ratio & $95 \% \mathrm{Cl}$ \\
\hline \multicolumn{3}{|l|}{ HPV infection } \\
\hline No & 3.3 & $(1.3-8.6)$ \\
\hline Yes & 1 & \\
\hline \multicolumn{3}{|c|}{ Number of lifetime sexual partners } \\
\hline$\leqslant 5$ & 4.1 & $(1.4-11.5)$ \\
\hline$>5$ & I & \\
\hline
\end{tabular}

$\mathrm{Cl}=$ confidence interval; HPV = human papillomavirus

repeat colposcopic examinations typically were not performed to confirm the persistence of CIN after the diagnostic biopsy. This lack of confirmation of persistent disease is important because many cervical lesions may regress after the initial diagnostic biopsy. All patients enrolled in our study had biopsy-proven and persistent CIN 2 or 3 lesions. Colposcopic examinations were performed 1 month after the initial biopsy to document disease persistence. Moreover, two pathologists agreed on the histologic diagnoses at the beginning and end of the study.

After all 93 patients were analysed in our study, we found that the regression rate of CIN 2 and 3 without conventional therapy was unexpectedly high at 52\%. Regression included 58\% of CIN 2 lesions and 47\% of CIN 3 lesions. In contrast, Ostor's (1993) 40year review of all reports that addressed the natural history of CIN found that the regression rates of CIN 2 and 3 averaged only 43 and $32 \%$, respectively. Unfortunately, some of these studies were hampered by a failure to confirm persistent disease after initial biopsy and inconsistent methods of determining grade of CIN. As one of the few reports on biopsy-proven and persistent CIN 2 or 3 disease, we found that the regression rates of high-grade lesions are unexpectedly high. These findings might influence the decision to routinely manage all patients with high-grade dysplasia by ablation, LEEP, or cone biopsy, particularly if undetectable levels of HPV are present.

The presence of cervical HPV DNA often is associated with cytologic and histologic changes of CIN. Indeed, up to $90 \%$ of women with CIN are HPV DNA positive depending on the diagnostic method used (Kiviat et al, 1992; Schiffman, 1992). In our analysis, women who lacked detectable HPV by PCR following the biopsy had a significantly greater chance of CIN regression compared to those with persistent HPV infections. This finding is consistent with previous studies that have shown that persistent HPV infections enhance the development and persistence of squamous intraepithelial lesions (Karlsson et al, 1995; Romney et al, 1997; Ho et al, 1998). Furthermore, our patients who initially tested positive for HPV and subsequently developed undetectable levels of HPV had higher rates of CIN regression (63\%) than patients with HPV persistence (41\%). Previous data have also shown that the resolution of HPV infection reflects the natural regression patterns of squamous intraepithelial lesions (Nasiell et al, 1986; Daling et al, 1996). Since most HPV infections are transient (Rosenfeld et al, 1992; Hsing et al, 1994; Hinchliffe et al, 1995; Romney et al, 1997), the patients in our study with high-grade lesions, who had no detectable HPV in their cervical scrapings following the biopsy, may have had a resolving HPV infection. Thus, based on our findings and that of other investigations, it appears that the resolution of HPV infection is predictive of the spontaneous regression of high-grade lesions. This has strong implications for the role of antiviral therapies against HPV and subsequent regression of high-grade dysplasia. Unlike previous reports (Campion et al, 1986; Kataja et al, 1990), our study did not find a significant difference in regression rates between patients with high risk $(16,18,31,33,35$, $39,45,51,52,56$, and 58) and other HPV types. However, we 
did find a lower rate of regression in patients infected with both low- and high-risk HPV types. Similarly, Ellerbrock et al (2000) also found that persistent HPV infection with or without type 16 or 18 was significantly associated with incident cervical lesions.

The role of HPV infection provides only a partial explanation for the high rate of spontaneous regression in our patient population. Sexual risk factors also have been recognised as causative determinants for CIN and cervical cancer. In our study, we found an inverse relationship between the number of sexual partners and CIN regression. Multivariate analyses of our study population showed that the effects of sexual risk factors persist even after controlling for HPV infections; suggesting that the presence of other sexually transmitted disease may also play a role in the natural history of CIN 2 or 3.

Our data propose that there may be selected patients with CIN who could be followed for short term in anticipation of spontaneous regression; this group includes those with no evidence of an HPV infection and low sexual risk factors. Indeed, the ASCUS-LSIL Triage Study reached this conclusion for patients with ASCUS Pap smears in whom HPV is not present (Solomon

\section{REFERENCES}

Bernard HU, Chan SY, Manos MM, Ong CK, Villa LL, Delius H, Peyton CL, Bauer HM, Wheeler CM (1994) Identification and assessment of known and novel human papillomaviruses by polymerase chain reaction amplification, restriction fragment length polymorphisms, nucleotide sequence, and phylogenetic algorithms. J Infect Dis 170: $1077-1085$

Campion MJ, McCance DJ, Cuzick J, Singer A (1986) Progressive potential of mild cervical atypia: prospective cytological, colposcopic, and virological study. Lancet 2: $237-240$

Daling JR, Madeleine MM, McKnight B, Carter JJ, Wipf GC, Ashley R, Schwartz SM, Beckmann AM, Hagensee ME, Mandelson MT, Galloway DA (1996) The relationship of human papillomavirus-related cervical tumors to cigarette smoking, oral contraceptive use, and prior herpes simplex virus type 2 infection. Cancer Epidemiol Biomarkers Prev 5: $541-548$

Ellerbrock TV, Chiasson MA, Bush TJ, Sun XW, Sawo D, Brudney K, Wright Jr TC (2000) Incidence of cervical squamous intraepithelial lesions in HIV-infected women. JAMA 283: 1031-1037

Flannelly G, Anderson D, Kitchener HC, Mann EM, Campbell M, Fisher P, Walker F, Templeton AA (1994) Management of women with mild and moderate cervical dyskaryosis [see comments]. BMJ 308: 1399-1403

Gregoire L, Arella M, Campione-Piccardo J, Lancaster WD (1989) Amplification of human papillomavirus DNA sequences by using conserved primers. J Clin Microbiol 27: 2660-2665

Harlan LC, Bernstein AB, Kessler LG (1991) Cervical cancer screening: who is not screened and why? Am J Public Health 81: 885-890

Herzog TJ, Williams S, Adler LM, Rader JS, Kubiniec RT, Camel HM, Mutch DG (1995) Potential of cervical electrosurgical excision procedure for diagnosis and treatment of cervical intraepithelial neoplasia. Gynecol Oncol 57: 286-293

Hillard PA, Biro FM, Wildey L (1991) Complications of cervical cryotherapy in adolescents. J Reprod Med 36: 711-716

Hinchliffe SA, van Velzen D, Korporaal H, Kok PL, Boon ME (1995) Transience of cervical HPV infection in sexually active, young women with normal cervicovaginal cytology. Br J Cancer 72: 943 - 945

Ho GY, Bierman R, Beardsley L, Chang CJ, Burk RD (1998) Natural history of cervicovaginal papillomavirus infection in young women. $\mathrm{N} \mathrm{Engl} \mathrm{J}$ Med 338: $423-428$

Holowaty P, Miller AB, Rohan T, To T (1999) Natural history of dysplasia of the uterine cervix. J Natl Cancer Inst 91: 252-258

Hsing AW, Schiffman M, Zhang T, Greer CE, Chen CJ, You SL, Hsieh CY, Huang TW, Liaw KL, Manos M (1994) Persistence of type-specific human papillomavirus infection among cytologically normal women [letter; comment]. J Infect Dis 170: 498

IARC (1995) IARC Monographs on the Evaluation of Carcinogenic Risks to Humans 64. Lyon, France: International Agency for Research on Cancer et al, 2001). Certainly, larger studies are needed to determine the safety and efficacy of conservative treatment of CIN 2 or 3; however, the short-term risk of progressing onto cancer in this population is very low (Holowaty et al, 1999). It is possible that the recommendation to observe women with ASCUS Pap smears who are negative for HPV may be applicable as short-term recommendations for women with established CIN, even when of high grade. Lastly, clinical trials are needed to determine the role of anti-HPV therapies including vaccines and immune modulators to prevent or eliminate HPV infection and potentially enhance the regression of CIN. With the high rate of spontaneous regression of CIN 2 and 3 in our patients, it may be safe and feasible to initiate a prospective, randomised trial to further investigate the natural regression of high-grade dysplasia.

\section{ACKNOWLEDGEMENTS}

We acknowledge Sandhya Upasani for her statistical support. This work was financially supported by National Cancer Institute, National Institutes of Health (NCI RO1-CA-50364).
Karlsson R, Jonsson M, Edlund K, Evander M, Gustavsson A, Boden E, Rylander E, Wadell G (1995) Lifetime number of partners as the only independent risk factor for human papillomavirus infection: a population-based study. Sex Transm Dis 22: 119-127

Kataja V, Syrjanen K, Syrjanen S, Mantyjarvi R, Yliskoski M, Saarikoski S, Salonen JT (1990) Prospective follow-up of genital HPV infections: survival analysis of the HPV typing data. Eur J Epidemiol 6: 9-14

Keefe KA, Schell MJ, Brewer C, McHale M, Brewster W, Chapman JA, Rose GS, McMeeken DS, Lagerberg W, Peng YM, Wilczynski SP, Anton-Culver H, Meyskens FL, Berman ML (2001) A randomized, double blind, Phase III trial using oral beta-carotene supplementation for women with highgrade cervical intraepithelial neoplasia. Cancer Epidemiol Biomarkers Prev 10: 1029-1035

Kiviat N (1996) Natural history of cervical neoplasia: overview and update. Am J Obstet Gynecol 175: 1099-1104

Kiviat NB, Koutsky LA, Critchlow CW, Lorincz AT, Cullen AP, Brockway J, Holmes KK (1992) Prevalence and cytologic manifestations of human papilloma virus (HPV) types $6,11,16,18,31,33,35,42,43,44,45,51,52$, and 56 among 500 consecutive women. Int J Gynecol Pathol 11: 197-203

Luthra UK, Prabhakar AK, Seth P, Agarwal SS, Murthy NS, Bhatnagar P, Das DK, Sharma BK (1987) Natural history of precancerous and early cancerous lesions of the uterine cervix. Acta Cytol 31: 226-234

Monk BJ, Cook N, Ahn C, Vasilev SA, Berman ML, Wilczynski SP (1994) Comparison of the polymerase chain reaction and Southern blot analysis in detecting and typing human papilloma virus deoxyribonucleic acid in tumors of the lower female genital tract. Diagn Mol Pathol 3: 283-291

Montz FJ, Monk BJ, Fowler JM, Nguyen L (1992) Natural history of the minimally abnormal Papanicolaou smear. Obstet Gynecol 80: 385-388

Murthy NS, Sardana S, Narang N, Agarwal SS, Sharma S, Das DK (1996) Biological behaviour of moderate dysplasia - a prospective study. Indian J Cancer 33: 24-30

Nasiell K, Nasiell M, Vaclavinkova V (1983) Behavior of moderate cervical dysplasia during long-term follow-up. Obstet Gynecol 61: 609-614

Nasiell K, Roger V, Nasiell M (1986) Behavior of mild cervical dysplasia during long-term follow-up. Obstet Gynecol 67: 665-669

Ostor AG (1993) Natural history of cervical intraepithelial neoplasia: a critical review. Int J Gynecol Pathol 12: 186-192

Ponten J, Adami HO, Bergstrom R, Dillner J, Friberg LG, Gustafsson L, Miller AB, Parkin DM, Sparen P, Trichopoulos D (1995) Strategies for global control of cervical cancer [see comments]. Int J Cancer 60: 1-26

Richart RM, Barron BA (1969) A follow-up study of patients with cervical dysplasia. Am J Obstet Gynecol 105: 386-393

Romney SL, Ho GY, Palan PR, Basu J, Kadish AS, Klein S, Mikhail M, Hagan RJ, Chang CJ, Burk RD (1997) Effects of beta-carotene and other factors on outcome of cervical dysplasia and human papillomavirus infection. Gynecol Oncol 65: 483-492 
Rosenfeld WD, Rose E, Vermund SH, Schreiber K, Burk RD (1992) Followup evaluation of cervicovaginal human papillomavirus infection in adolescents. J Pediatr 121: 307-311

Schiffman MH (1992) Recent progress in defining the epidemiology of human papillomavirus infection and cervical neoplasia. J Natl Cancer Inst 84: $394-398$

Schiffman MH, Brinton LA (1995) The epidemiology of cervical carcinogenesis. Cancer 76: 1888-1901

Solomon D, Schiffman M, Tarone R (2001) Comparison of three management strategies for patients with atypical squamous cells of undetermined significance: baseline results from a randomized trial. $J$ Natl Cancer Inst 93: 293-299

Syrjanen K, Kataja V, Yliskoski M, Chang F, Syrjanen S, Saarikoski S (1992) Natural history of cervical human papillomavirus lesions does not substantiate the biologic relevance of the Bethesda System. Obstet Gynecol 79: 675-682 